\title{
INFLUÊNCIA DA DEMOGRAFIA SOBRE A CONSCIÊNCIA AMBIENTAL E CONSUMO ECOLÓGICO
}

\author{
INFLUENCE OF DEMOGRAPHY ON ENVIRONMENTAL AWARENESS AND ECOLOGICAL
} CONSUMPTION

Recebido em 28.05.2017. Aprovado em 05.09.2017

Avaliado pelo sistema double blind review

DOI: http://dx.doi.org/10.12712/rpca.v11i4.990

\author{
Bianca Cristina Longo \\ Universidade Estadual do Oeste do Paraná (UNIOESTE), Cascavel/PR, BRASIL \\ biancalongo1@hotmail.com
}

\section{Ivano Ribeiro}

Universidade Estadual do Oeste do Paraná (UNIOESTE), Cascavel/PR, BRASIL ivano.adm@gmail.com

\section{Antonio Oliveira de Carvalho}

Universidade Nove de Julho (UNINOVE), São Paulo/SP, BRASIL

professorcarvalho@rocketmail.com

\section{Geysler Rogis Flor Bertolini}

Universidade Nove de Julho (UNINOVE), São Paulo/SP, BRASIL

professorcarvalho@rocketmail.com

\begin{abstract}
Resumo
Este artigo teve como objetivo analisar as influências dos fatores demográficos sobre o grau de percepção da consciência ambiental e do consumo ecológico. A partir de dados decorrentes de 1075 questionários respondidos foram testadas seis hipóteses de pesquisa. As variáveis independentes foram: sexo, idade e escolaridade. Os resultados demonstram que o sexo feminino e a idade mais elevada estão relacionadas de forma positiva e significante com o maior grau de percepção da consciência ambiental e do consumo ecológico. Já a escolaridade está positivamente relacionada com o maior grau de percepção da consciência ambiental, porém, sem efeito significativo sobre a percepção do consumo ecológico. Como grande parte dos estudos que investigaram este tema se concentram em aspectos comportamentais, a principal contribuição desse estudo é o melhor entendimento sobre o efeito das variáveis demográficas sobre a percepção dos indivíduos quanto ao grau de consciência ambiental e consumo ecológico.
\end{abstract}

Palavras-chave: Sustentabilidade; Meio ambiente; Características dos consumidores.

\begin{abstract}
This article aimed to analyze the influence of demographic factors on the degree of perception of environmental awareness and ecological consumption. Based on data from 1075 questionnaires answered, six hypotheses were tested. The independent variables were: gender, age and educational level. The results show that female and older age are positively and significantly related to the degree of environmental awareness and ecological consumption. The variable education is positively related to the degree of perception of environmental awareness, but without significant effect on the perception of ecological consumption. As a large part of the studies that investigate this theme concentrate on behavioral aspects, the main contribution of this study is the better understanding about the effect of the demographic variables on the individuals' perception regarding the degree of environmental awareness and ecological consumption.
\end{abstract}

Keywords: Sustainability; Environment; Characteristics of consumers. 


\section{Introdução}

Os estudos que envolvem aspectos ligados com a sustentabilidade ganham cada vez mais espaço, conforme destacam Peçanha e lizuka (2014) e Dal Ben et al., (2017). Contudo, ainda existem questionamentos sobre as variáveis que podem se relacionar com uma maior consciência ambiental e o consumo ecologicamente correto. Diversos destas investigações se pautam na análise de variáveis de fundo comportamental (CÔRTES et al., 2016; LARSON et al., 2015; LEVINE; STRUBE, 2012; STEG; VLEK, 2009). Spínola (2001) também destaca que a ética e os valores individuais podem ser influenciadores de um modo de vida mais sustentável.

Além do entendimento sobre os aspectos comportamentais, outras questões ligadas com as variáveis demográficas dos indivíduos, e como estas variáveis podem afetar a conduta e o comportamento em relação ao meio ambiente, ainda são pontos que carecem de investigações (BALDERJAHN, 1988; DI CIOMMO, 2003; EAGLY, 1987; OKAMOTO, 1996; SOARES, 1998; STERN; DIETZ; KALOF, 1993; TORNQUIS; LISBOA; MONTYSUMA, 2010). A partir destes argumentos este estudo se pauta na seguinte questão de pesquisa: quais as influências dos fatores demográficos sobre o grau de percepção de consciência ambiental e de consumo ecológico?

Para a realização do estudo utilizou-se dados de 1075 questionários que foram respondidos entre os meses de junho e agosto de 2015. Foi utilizado o Instrumento de Mensuração do Grau de Consciência Ambiental, do Consumo Ecológico e dos Critérios de Compra dos Consumidores, desenvolvido e validado por Bertolini e Possamai (2005). Os dados foram tratados de forma quantitativa, sendo possível testar seis hipóteses de pesquisa relacionadas com as duas variáveis dependentes - consciência ambiental e consumo ecológico.

Os resultados apresentados por meio de modelos de regressão linear, contemplam ainda, duas variáveis de controle - renda, e a região de moradia. Verifica-se que o grau de percepção de consciência ambiental é influenciado de forma positiva e significante pelo: sexo, idade e o nível de escolaridade. Já o consumo ecológico não foi afetado pelo nível de escolaridade.

O estudo é apresentado em sete seções incluindo esta introdução; a segunda se destina ao referencial teórico; na terceira são formuladas as hipóteses de pesquisa; a quarta é referente ao método utilizado; na quinta apresenta-se os resultados de pesquisa; na sexta é efetuada a discussão dos resultados encontrados; já a sétima e última seção é destinada para a conclusão do estudo, com foco para apresentação das contribuições do artigo.

\section{Referencial teórico}

Nesta seção é apresentado um quadro teórico relativo a meio ambiente, desenvolvimento sustentável, consciência ambiental, consumo ecológico e fatores demográficos. A partir da compreensão destes elementos é apresentado na seção seguinte o modelo proposto e as hipóteses a serem testadas na pesquisa.

\section{Meio ambiente e Desenvolvimento Sustentável}

Segundo Pereira e Curi (2012) a medida que estudos sobre o meio ambiente se desenvolvem, emergem várias percepções culturais, afinal, o meio ambiente constitui-se em componente básico da própria existência social. Ferreira (2013) e Carvalho et al. (2015) destacam que a preservação do meio ambiente é uma responsabilidade de todos, pois deve atender as necessidades atuais, e possa ser repassada para as próximas gerações.

Assim, diversas são as formas e compromissos em prol da sustentabilidade, entre elas, pode-se citar os compromissos assumidos na Rio+20 sobre o desenvolvimento sustentável, "prometendo promover um futuro econômico, social e ambientalmente sustentável para o nosso planeta e para as gerações do presente e do futuro. Diversos países também reafirmaram os princípios enunciados na Cúpula da Terra de 1992 e em diversas conferências mundiais sobre desenvolvimento sustentável” (ONU, 2010).

Para Brüseke (1995) havendo a continuidade dos atuais níveis de: crescimento populacional, industrialização, poluição, produção e redução dos recursos naturais, os limites do planeta serão alcançados nos próximos cem anos, resultando no provável declínio súbito dos recursos naturais e consequentemente da população e da capacidade industrial. Para Kates et al. (2005) o planeta vive uma situação insustentável, pois, os ecossistemas que suportam a vida apresentam níveis de degradação elevados, e ainda são observados como fonte de geração econômica pelos seus diversos públicos de interesse. 
De acordo com Jacobi (2003) para uma reflexão acerca de práticas e atitudes em um contexto em que o meio ambiente se encontra parcialmente degradado, se carece de um - universo educativo, que é a articulação de diversos atores em prol do meio ambiente. Para o autor é necessária a potencialização dos sistemas de geração de conhecimento em uma perspectiva multidisciplinar, o que poderia ser desenvolvido a partir da capacitação de profissionais das mais diversas áreas do conhecimento.

Para que o desenvolvimento ocorra a sociedade deve criar hábitos mais conscientes perante o meio ambiente, como o de consumo mais adequado (BRANDALISE et al., 2014; MOREIRA, 2015). O meio ambiente implica na educação para um consumo consciente, além de estimular o exercício da resolução de problemas reais para concretização de projetos que visam a preveni-los. Neste sentido o que se observa é um aprendizado e respeito de toda a diversidade, riqueza e a complexidade ambiental (REIS; SEMÊDO; GOMES, 2012).

Contudo, não se pode analisar questões relativas ao meio ambiente sem considerar um amplo contexto no qual este tema está inserido. Mendes (2009) ao abordar as dimensões de sustentabilidade - dimensão social, dimensão econômica e dimensão ambiental destaca que todas estão interligadas e são responsáveis pelas mudanças de comportamento dos indivíduos, portanto, não se pode isola-las.

\section{Consciência ambiental e consumo ecológico}

O processo de decisão de compra de produtos ecológicos é composto por estágios que estão relacionados com as necessidades, desejos e a percepção do consumidor sobre aquilo que é ofertado (LOPES; PACAGNAN, 2014; SILVA; LOPES, 2015). Autores como Engel, Blackwell e Miniard (1995), Solomon (1996) e Schiffman e Kanuk (2000) consideram ainda uma série de etapas no processo de compra, sendo: reconhecimento de necessidades, busca de informações, avaliação de alternativas, decisão de compra e comportamento pós-compra.

Na visão de Soares, Navarro e Ferreira (2004) conforme se disseminou o debate sobre a complexidade que envolve produção de bens e o meio ambiente, as organizações responderam ao apelo dos consumidores e começaram a valorizar os aspectos ambientais ligados aos seus produtos e formas produtivas. Esta - ética ambiental - deve considerar a saúde, a educação, a qualidade de vida, o direito individual, a política e cultura, requerendo uma articulação precisa com valores e justiça social.

A valorização destes elementos promoveu discussões sobre a conscientização ambiental e da necessidade de mudanças significativas em relação ao zelo pelos recursos naturais (SOARES; NAVARRO;FERREIRA, 2004). Segundo Moisander (2007) as decisões diárias de compra colocam o consumidor diante de um tradeoff geralmente conflitante entre o resultado de uma complexidade motivacional e a prática do consumo verde. Young et al. (2010) ressaltam que, em cada decisão de compra de um produto ou serviço, existe o potencial de - maior ou menor - contribuição para um padrão de consumo sustentável. Para os autores, cada compra tem em si: ética, recursos, resíduos e impacto ambiental. Assim, quando o indivíduo considera as questões ambientais nas decisões, todo o processo torna-se ainda mais complexo.

Esta consciência de se consumir de forma mais sustentável se relaciona com o conhecimento de cada um sobre a necessidade da manutenção dos recursos naturais para futuras gerações (REIS; SEMÊDO; GOMES, 2012). Para Bertolini e Possamai, (2005) a consciência ambiental é um fator determinante para um comportamento ecológico. Ela propicia uma maior facilidade na identificação da melhor forma de adaptação de consumo frente aosncuidados a serem tomados com o meio ambiente.

De acordo com Camponogara, Ramos e Kirchhof (2009) refletir sobre o próprio comportamento e obter o conhecimento sobre os problemas relacionados ao meio ambiente, é algo que se deve obter através de uma estratégia, e estar convicto das atitudes a serem tomadas. Para o autor, formas de apropriação do conhecimento e ampla divulgação sobre o tema criam um desenvolvimento de ações para preservação ambiental

Diversos são os movimentos de organizações que se propõem em melhorar suas ações em prol do meio ambiente (PEREIRA; CURI, 2012). Porém, é necessário que a sociedade como um todo possua acesso à informação e formação sobre os impactos ambientais ocasionados pelo consumo dos mais diversos produtos. Hames, Frizon e Araujo (2009) colocam que a maior interação do sujeito com o meio ambiente poderá suscitar reflexões sobre esta relação homem x natureza. 
Percebe-se que, a discussão sobre a melhoria do grau de consciência ambiental é algo recente (PEREIRA; CURI, 2012). De acordo com Iserhardt et al. (2009) o aumento deste grau depende de um processo de educação, e que deve ser inserido em todos os contextos da atuação humana e na sociedade do consumo.

Para Connelly, Ketchen Jr e Slater (2011) estes aspectos ambientais, ainda são negligenciados, se constata a existência de uma desconexão entre o comportamento do consumidor e o da oferta de produtos ecológicos - produtos verdes - visto que estes ainda representam um percentual mínimo da oferta geral de produtos no mercado. Isto só deve mudar a partir de uma nova consciência da população em relação ao próprio padrão de consumo.

\section{Demografia e meio ambiente}

O entendimento sobre os padrões de consumo, e compressão sobre o comportamento dos consumidores de produtos ecologicamente corretos ainda é escasso. Ainda não se pode generalizar estes grupos, mas os adeptos ao consumo consciente podem ser vistos como minoria. Um exemplo disso, conforme Gorni, Gomes e Dreher (2012), está nos produtos tecnológicos com recorde de alterações, como celulares, computadores, entre outros. Os autores constataram que há uma tentativa de adaptação destas novidades entre os diferentes grupos: jovens, homens ou mulheres. Os hábitos de consumo podem variar quanto ao gênero, considerando-se que homens e mulheres possuem necessidades que se distinguem entre si. Os autores destacam que essas diferenças devem ser consideradas e avaliadas na realização de estudos acerca do consumo sustentável.

A idade e o nível de escolaridade são variáveis de grande importância ao se investigar o meio ambiente. Padrões de consumo de crianças e jovens podem se refletir para o restante da vida adulta, assim como, conhecimentos adquiridos por meio da educação podem interferir diretamente nas decisões de consumo. Logo, indivíduos que são estimulados ao consumo mais sustentável poderão gerar um impacto reduzido no meio ambiente (COSTA, 2011).

A preocupação com o desenvolvimento da consciência ambiental é vista há muito tempo como uma forma de garantir um futuro mais sustentável. O Relatório Brundtland (1987) cita que é necessário satisfazer as necessidades do presente, sem comprometer a capacidade de as gerações vindouras satisfazerem as suas próprias necessidades. Para que isso ocorra, a educação possui um papel central, podendo explorar conteúdos que visem disseminar informações sobre a importância de uma consciência e consumo mais adequado em relação ao meio ambiente. Para Jacobi (2003, p.192), a desresponsabilização da população decorre "principalmente da desinformação, da falta de consciência ambiental e de um déficit de práticas comunitárias baseadas na participação e no envolvimento dos cidadãos".

Dada uma perspectiva de consumo relacionada com gênero, idade e escolaridade, padrões de consumo podem ser determinados pelos comportamentos habituais dos indivíduos. Contudo, estes comportamentos podem sofrer interferências de alguns fatores demográficos, conforme destacados acima. Na seção seguinte é apresentado o modelo de pesquisa e as hipóteses formuladas.

\section{Hipóteses e modelo de pesquisa}

Os estudos que investigam as relações do público feminino com os aspectos sociais e ambientais indicam que as mulheres possuem um alto grau de consciência ambiental (DI CIOMMO, 2003). Para o autor este maior interesse pelo consumo consciente por parte das mulheres ocorre desde o início dos anos 90 em decorrência do movimento ecofeminista.

Para Soares (1998) o ecofeminismo incorporou às discussões ambientais uma perspectiva inovadora, onde aspectos que não eram vistos, até então, como economicamente relevantes, como: a cultura local; qualidade de vida; e os valores da população, começaram a ser observados a partir deste movimento. Assim, a própria presença das mulheres junto à movimentos relacionados ao meio ambiente também cresceu, e elas se tornaram protagonistas de ações e políticas ambientais (TORNQUIS; LISBOA; MONTYSUMA, 2010).

Da mesma forma que o grau de consciência ambiental, o consumo ecológico também pode ser influenciado pelo sexo. Estudos de Eagly (1987); Stern, Dietz e Kalof (1993); Laroche, Bergeron e Barbaro-Forleo (2001); Di Ciommo (2003) e Paço e Raposo (2010) sugerem a existência de uma forte correlação entre 
comportamento feminino com ações ligadas com o meio ambiente. A partir destas observações foi formulado o primeiro conjunto de hipóteses de pesquisa:

Hipótese 1a. Existe uma influência positiva e significante do sexo feminino sobre o grau de consciência ambiental.

Hipótese 1b. Existe uma influência positiva e significante do sexo feminino sobre o grau de consumo ecológico.

Mesmo com a difusão sobre a necessidade de uma maior consciência ambiental por parte da população mundial, Bonnet et al. (2002) apontam que as populações mais jovens ainda necessitam desenvolver um comportamento mais proativo em relação ao meio ambiente. Os autores identificaram que em uma região universitária e com grande concentração de jovens na França, os consumos de energia, água e de outros serviços ofertados, eram relativamente altos quando comparados com a média das grandes metrópoles. Isto sugere que, mesmo inseridos em um contexto de geração de conhecimentos as ações dos jovens ainda estão aquém do ideal.

Como a consciência é que leva ao comportamento, conforme destaca Okamoto (1996), o comportamento dos jovens sugere a existência de um menor grau de consciência ambiental em relação aos mais velhos. Pessoas mais velhas possuem um maior nível de experiência, desta forma, possuem um maior entendimento e uma relação mais forte com questões ambientais (STEGER; MEIMA, 1998). Indivíduos com idade mais elevadas também parecem ser mais propensos a se envolverem com ações ambientais, como: evitar danos ambientais e consumo mais consciente de matérias-primas e recursos naturais. Assim, pode-se elaborar o segundo conjunto de hipóteses de pesquisa (BRENTON; DENIZ; STEPHAN, 2013).

Hipótese 2a. Existe uma influência positiva e significante da idade mais elevada sobre o grau de consciência ambiental.

Hipótese 2b. Existe uma influência positiva e significante da idade mais elevada sobre o grau de consumo ecológico.
Algumas outras variáveis podem estar relacionadas diretamente com o grau de consciência ambiental. $\mathrm{Na}$ visão de Balderjahn (1988) os indivíduos mais preocupados com as questões ambientais são aqueles que possuem um maior grau de educação. A Educação possui um papel contribuir central na mudança de uma realidade, sendo capaz de alterar e criar comportamentos mais sustentáveis (GUIMARÃES et al., 2009).

A educação também é uma das principais ferramentas relacionadas com as resoluções de problemas com o meio ambiente. Ela proporciona uma melhor visão sobre a realidade deste sistema dinâmico que é a relação entre desenvolvimento e meio-ambiente (MARIGA, 2006). Para Jabbour (2014) a deterioração do meio ambiente pode retroceder a partir do momento que os indivíduos possuem conhecimento e atitudes pró gestão do meio ambiente, e isto pode ocorrer por meio da educação formal. Diante destas observações, podese inferir que a escolaridade direciona para uma maior consciência sobre a importância do meio ambiente, e assim, formula-se o terceiro conjunto de hipóteses deste estudo.

Hipótese 3a. Existe uma influência positiva e significante do nível de escolaridade sobre grau de consciência ambiental.

Hipótese 3b. Existe uma influência positiva e significante do nível de escolaridade sobre grau de consumo ecológico.

A Figura 1 apresenta o modelo de pesquisa, onde o grau de consciência ambiental e o consumo ecológico são as variáveis dependentes, sendo o sexo, idade e a escolaridade as variáveis independentes. As variáveis de controle foram a renda e a região de residência. De acordo com as hipóteses formuladas todas as relações entre as variáveis independentes e dependentes são positivas. 
Figura 1. Modelo de pesquisa.

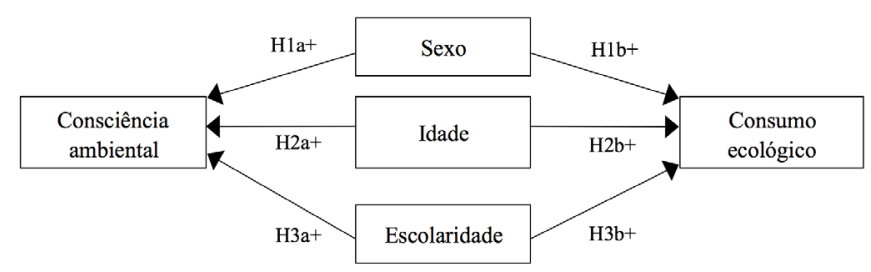

Fonte: Elaborado pelos autores (2017).

Na próxima seção destaca-se o método de mensuração e operacionalização destas variáveis, assim como, as informações sobre o instrumento de pesquisa, a amostra escolhida e a forma de análise e apresentação dos resultados.

\section{Método}

\section{Variáveis dependentes}

As variáveis dependentes deste estudo foram o grau de consciência ambiental e o consumo ecológico, para esta identificação optou-se em mensurar cada variável por meio da percepção dos respondentes. Foi utilizado o Instrumento de Mensuração do Grau de Consciência Ambiental, do Consumo Ecológico e dos Critérios de Compra dos Consumidores de Bertolini e Possamai (2005). Neste instrumento, os autores apresentam uma lista de 16 questões medidas por meio de uma escala de 4 pontos, que são capazes de mensurar ambas variáveis investigadas, conforme Quadro 1.

Quadro 1. Instrumento de mensuração do grau de consciência ambiental

\begin{tabular}{|c|c|}
\hline & $\begin{array}{c}\text { stões para mensuração do grau de consciência } \\
\text { ambiental }\end{array}$ \\
\hline & $\begin{array}{l}\text { Antes de jogar algo no lixo, você pensa em como } \\
\text { poderia reutilizá-lo? }\end{array}$ \\
\hline 2 & $\begin{array}{l}\text { Você separa o lixo que pode ser reciclado, como o } \\
\text { papel, plástico, alumínio, vidro ou metais ferrosos? }\end{array}$ \\
\hline 3 & $\begin{array}{l}\text { Evita a queima de lixo doméstico (plásticos, isopor, } \\
\text { restos orgânicos)? }\end{array}$ \\
\hline 4 & $\begin{array}{l}\text { Procura não deixar a torneira aberta ao escovar os } \\
\text { dentes ou ao fazer a barba? }\end{array}$ \\
\hline 5 & Apaga as luzes e TV quando sai do ambiente? \\
\hline & $\begin{array}{l}\text { Utiliza máquinas de lavar roupas ou louças apenas } \\
\text { quando estiverem com capacidade máxima } \\
\text { preenchida? }\end{array}$ \\
\hline
\end{tabular}

7 Você se preocupa em não jogar lixo na rua?

8 Você utiliza os dois lados dos papéis, ou reutiliza rascunhos?

Questões para mensuração do consumo ecológico

1 Ao comprar, você tem a prática de procurar saber se o fabricante tem ações ambientais (leva em conta a postura ambiental do fabricante antes de comprar)?

2 Tem a prática de comprar produtos e embalagens fabricados com material reciclado ou que podem ser recicláveis?

3 Antes da compra, você tem a prática de verificar rótulos e embalagens para identificar um produto ambientalmente correto?

$4 \quad$ Você tem a prática de comprar produtos orgânicos?

$5 \quad$ Você tem a prática de comprar produtos de limpeza biodegradáveis?

6 Você tem a prática de comprar lâmpadas e eletrodomésticos que gastam menos energia?

7 Você tem a prática de pagar mais por um produto que não polui o meio ambiente?

8 Nas compras, ao encontrar um produto com rótulo que informa que ele foi fabricado de maneira ambientalmente correta, você fica motivado em compra-lo?

Fonte: Bertolini e Possamai (2005).

A adoção deste instrumento se deve ao entendimento que este é adequado aos objetivos deste artigo, já que foi desenvolvido e validado ao contexto brasileiro. Destaca-se que, devido às especificidades das análises quantitativas efetuadas neste estudo, a escala original foi invertida, sendo utilizado: (1) nunca pratico, (2) pratico pouquíssimas vezes, (3) pratico algumas vezes, e (4) pratico sempre. O grau de consciência ambiental foi a média simples das oito questões do instrumento. A escala também apresentou consistência interna (Alpha de Cronbach > 0,70), reforçando o suporte para sua utilização.

\section{Variáveis independentes e de controle}

Foram analisadas três variáveis independentes, a variável sexo que foi codificada em variável dicotômica: (0) feminino e (1) masculino; a idade dos participantes também codificada em: (1) até 24 anos, (2) entre 25 e 34 anos, (3) entre 35 e 49 anos, e (4) 50 anos ou mais; e a escolaridade que foi medida em: (1) ensino fundamental, (2) ensino médio, (3) ensino superior, (4) especialização, e (5) mestrado/doutorado. 
Foram inseridas ainda duas variáveis de controle, a renda dos participantes e a região onde reside, a primeira renda foi medida pela - renda familiar, sendo: (1) até 1 salário mínimo, (2) entre 1 e 5 salários mínimos, (3) entre 5 e 10 salários mínimos, (4) entre 10 e 15 salários mínimos, e (5) para os que tivessem 15 ou mais salários mínimos. Já região foi codificada em uma variável dicotômica, sendo: (0) região central e (1) bairros e periferia do município pesquisado. Estas duas variáreis tiveram o objetivo de controlar o modelo, já que, ambas podem exercer influências sobre os fatores investigados (SEQUINEL; CARON, 2010).

\section{Coleta e análise dos dados}

A amostra foi composta por 1075 respondentes de um questionário aplicado, conforme a Tabela 1. A aplicação foi realizada de forma eletrônica por meio de formulário do Google Forms e também de forma impressa. Devido as particularidades de cada município, que podem possuir diferenças em termos de renda e moradia em função de residir em área central ou periferia, o estudo foi delimitado para os moradores do município de Cascavel, Paraná - Brasil.

Tabela 1. Abrangência de questionários respondidos.

\begin{tabular}{l|c|c}
\hline \multicolumn{1}{c|}{ Região } & Questionários Respondidos & Percentual \\
\hline Central & 344 & $32 \%$ \\
\hline Bairros/periferia & 731 & $68 \%$ \\
\hline TOTAL & $\mathbf{1 0 7 5}$ & $100 \%$ \\
\hline
\end{tabular}

Fonte: Elaborado pelos autores (2017).

Após coletados, os dados foram analisados de forma quantitativa, inicialmente foi realizada uma análise descritiva de correlações e posteriormente, por meio de modelos de regressão linear, foram testadas as hipóteses de pesquisa. Para atender os pressupostos desta última análise, verificou-se a existência de multicolinearidade entre as variáveis independentes, onde um valor de VIF (Variance Inflation Factor) máximo de 1,252 confirmou a possibilidade da utilização da técnica de regressão.

\section{Resultados}

Inicialmente foi realizada uma análise descritiva dos dados. As informações referentes às variáveis pesquisadas são apresentadas na Tabela 02, destacando-se a média, desvios padrão e correlações de Pearson.

Tabela 2. Análises descritivas das variáveis de pesquisa

\begin{tabular}{clccccccccc}
\hline $\mathbf{N}$ & Variáveis & Média & SD & $\mathbf{1}$ & $\mathbf{2}$ & $\mathbf{3}$ & $\mathbf{4}$ & $\mathbf{5}$ & $\mathbf{6}$ & $\mathbf{7}$ \\
\hline $\mathbf{1}$ & Renda familiar & 2,48 & 0,92 & 1 & & & & & & \\
$\mathbf{2}$ & Região & 0,15 & 0,36 &, $225^{* *}$ & 1 & & & & & \\
$\mathbf{3}$ & Sexo & 0,39 & 0,49 &, $162^{* *}$ &, $100^{* *}$ & 1 & & & \\
$\mathbf{4}$ & Idade & 1,66 & 0,99 & $-0,037$ & $-0,029$ &,$- 196^{* *}$ & 1 & & \\
$\mathbf{5}$ & Escolaridade & 2,33 & 0,89 &, $200^{* *}$ & 0,046 &,$- 122^{* *}$ &, $402^{* *}$ & 1 & & \\
$\mathbf{6}$ & Consciência & 3,26 & 0,47 & $-0,032$ & $-0,054$ &,$- 233^{* *}$ &, $423^{* *}$ &, $301^{* *}$ & 1 & \\
$\mathbf{7}$ & Consumo & 2,45 & 0,62 &,$- 066^{*}$ & $-0,059$ &,$- 169^{* *}$ &, $364^{* *}$ &, $157^{* *}$ &, $543^{* *}$ & 1 \\
\hline
\end{tabular}

**. Correlation is significant at the 0.01 level (2-tailed).

*. Correlation is significant at the 0.05 level (2-tailed).

Fonte: Elaborado pelos autores (2017). 
As análises descritivas por meio das médias, desvio padrão e correlações de Pearson, demonstram que existem correlações significantes, tanto da consciência ambiental, como do consumo ecológico com as três variáveis independentes. As variáveis idade e escolaridade apresentam uma correlação positiva com as variáveis dependentes, indicando que indivíduos com idade mais elevada e com maior escolaridade possuem uma relação positiva com o maior grau de consciência ambiental e consumo ecológico. Já as correlações negativas entre o sexo com as variáveis dependentes indicam que as mulheres possuem um maior grau de consciência ambiental e consumo ecológico do que os homens.

A partir destas observações, foram elaborados cinco modelos de regressão linear para cada uma das variáveis dependentes, e buscam confirmar ou refutar as hipóteses de pesquisa. No primeiro modelo estão presentes apenas as duas variáveis de controle - renda e região. Os modelos dois, três e quatro testam isoladamente as três variáveis independentes - sexo, idade e escolaridade. Já o quinto modelo possui todas as variáveis pesquisadas, sendo este, utilizado como critério final de análise, conforme Tabela 3.

Tabela 3. Modelos de regressões da variável dependente consciência ambiental

\begin{tabular}{|c|c|c|c|c|c|c|}
\hline Variáveis & & $\begin{array}{c}\text { Modelo } \\
1 \\
\end{array}$ & $\begin{array}{c}\text { Modelo } \\
2 \\
\end{array}$ & $\begin{array}{c}\text { Modelo } \\
3 \\
\end{array}$ & $\begin{array}{c}\text { Modelo } \\
4 \\
\end{array}$ & $\begin{array}{c}\text { Modelo } \\
5 \\
\end{array}$ \\
\hline \multicolumn{7}{|l|}{ Variáveis de controle } \\
\hline Renda & & $\begin{array}{l}-0,022 \\
(-, 687)\end{array}$ & $\begin{array}{l}0,016 \\
(, 503)\end{array}$ & $\begin{array}{l}-0,007 \\
(-, 238)\end{array}$ & $\begin{array}{l}-0,080^{*} \\
(-2,554)\end{array}$ & $\begin{array}{l}-0,011 \\
(-, 379)\end{array}$ \\
\hline Região & & $\begin{array}{r}-0,044 \\
(-1,367)\end{array}$ & $\begin{array}{l}-0,028 \\
(-, 893)\end{array}$ & $\begin{array}{r}-0,038 \\
(-1,311)\end{array}$ & $\begin{array}{r}-0,047 \\
(-1,539)\end{array}$ & $\begin{array}{r}-0,031 \\
(-1,084)\end{array}$ \\
\hline \multicolumn{7}{|l|}{ Variáveis independentes } \\
\hline Sexo & & & $\begin{array}{l}-0,251 * \\
(-8,155)\end{array}$ & & & $\begin{array}{l}-0,163^{*} \\
(-5,665)\end{array}$ \\
\hline Idade & & & & $\begin{array}{r}0,421 * \\
(14,825)\end{array}$ & & $\begin{array}{r}0,333^{*} \\
(10,928)\end{array}$ \\
\hline \multirow[t]{4}{*}{ Escolaridade } & & & & & $\begin{array}{r}0,307^{*} \\
(10,078)\end{array}$ & $\begin{array}{l}0,143^{*} \\
(4,625)\end{array}$ \\
\hline & $\mathrm{R}^{2}$ & 0,003 & 0,064 & 0,180 & 0,093 & 0,225 \\
\hline & $\mathrm{R}^{2}$ Ajustado & 0,001 & 0,061 & 0,178 & 0,090 & 0,221 \\
\hline & $\mathrm{F}$ & 1,456 & $23,202 *$ & $74,450 *$ & $34,798^{*}$ & $58,839^{*}$ \\
\hline
\end{tabular}

* Significante ao nível de 0,05

Valores t entre parênteses

Fonte: Elaborado pelos autores (2017).

A Tabela 3 apresenta os resultados dos testes estatísticos de regressão linear. A hipótese 1a sugeriu uma influência positiva entre o sexo feminino e o grau de consciência ambiental. Como foi utilizada a variável dicotomizada $(0)$ mulheres e (1) para os homens, o modelo 2 e modelo 5 permitem confirmar esta relação, por meio dos valores $(\beta=-0,251, \mathrm{p}<0,05)$ e $(\beta=-0,163, \mathrm{p}<0,05)$.

A hipóteses 2 a propôs a existência de uma influência positiva entre idade e o grau de consciência ambiental. A partir dos resultados $(\beta=0,421, \mathrm{p}<0,05)$ do modelo 3 e $(\beta=0,333, \mathrm{p}<0,05)$ do modelo 5 é possível confirmar esta hipótese, pois observou-se que os indivíduos mais velhos possuem uma influência positiva sobre o grau de consciência ambiental. Com um valor $(\beta=0,307, \mathrm{p}<0,05)$ no modelo 4 e $(\beta=0,143, \mathrm{p}<0,05)$ do modelo 5 também se pode confirmar a hipótese 3 a, no qual indica que um maior nível de escolaridade influencia de forma positiva o grau de consciência ambiental. No modelo 5, onde foram inseridas todas as variáveis de pesquisa, 
observa-se um poder de explicação (22\%), conforme $\mathrm{R}^{2}$ ajustado. Para testar o segundo conjunto de hipóteses as mesmas variáveis independentes e de controle forma utilizadas nos cinco modelos de regressão para testar o efeito sobre a variável consumo ecológico, conforme Tabela 4.

Tabela 4. Modelos de regressões da variável dependente consumo ecológico

\begin{tabular}{|c|c|c|c|c|c|}
\hline Variáveis & $\begin{array}{c}\text { Modelo } \\
1\end{array}$ & $\begin{array}{c}\text { Modelo } \\
2\end{array}$ & $\begin{array}{c}\text { Modelo } \\
3\end{array}$ & $\begin{array}{c}\text { Modelo } \\
4\end{array}$ & $\begin{array}{c}\text { Modelo } \\
5\end{array}$ \\
\hline \multicolumn{6}{|l|}{ Variáveis de controle } \\
\hline Renda & $\begin{array}{r}-0,057 \\
(-1,788)\end{array}$ & $\begin{array}{l}-0,031 \\
(-, 969)\end{array}$ & $\begin{array}{r}-0,044 \\
(-1,488)\end{array}$ & $\begin{array}{l}-0,088^{*} \\
(-2,731)\end{array}$ & $\begin{array}{l}-0,030 \\
(-, 979)\end{array}$ \\
\hline Região & $\begin{array}{l}-0,039 \\
(-, 039)\end{array}$ & $\begin{array}{l}-0,028 \\
(-, 894)\end{array}$ & $\begin{array}{r}-0,036 \\
(-1,191)\end{array}$ & $\begin{array}{r}-0,039 \\
(-1,249)\end{array}$ & $\begin{array}{l}-0,027 \\
(-, 920)\end{array}$ \\
\hline \multicolumn{6}{|l|}{ Variáveis independentes } \\
\hline Sexo & & $\begin{array}{l}-0,173^{*} \\
(-5,535)\end{array}$ & & & $\begin{array}{l}-0,102^{*} \\
(-3,369)\end{array}$ \\
\hline Idade & & & $\begin{array}{r}0,368^{*} \\
(12,651)\end{array}$ & & $\begin{array}{r}0,341^{*} \\
(10,660)\end{array}$ \\
\hline Escolaridade & & & & $\begin{array}{l}0,176^{*} \\
(5,610)\end{array}$ & $\begin{array}{l}0,020 \\
(, 629)\end{array}$ \\
\hline $\mathrm{R}^{2}$ & 0,006 & 0,035 & 0,141 & 0,035 & 0,151 \\
\hline $\mathrm{R}^{2}$ Ajustado & 0,004 & 0,032 & 0,139 & 0,032 & 0,147 \\
\hline $\mathrm{F}$ & 3,006 & $12,273^{*}$ & $55,698^{*}$ & $12,255^{*}$ & $36,018^{*}$ \\
\hline
\end{tabular}

* Significante ao nível de 0,05

Valores t entre parênteses.

Fonte: Elaborado pelos autores (2017).

Como utilizou-se a variável dicotomizada (0) mulheres e (1) para os homens, o modelo 2 e modelo 5 permite aceitar a hipótese $1 \mathrm{~b}(\beta=-0,173, \mathrm{p}<0,05)$ e $(\beta=-0,102, \mathrm{p}<0,05)$. Confirmando a existência de relação positiva e significante entre o sexo feminino e o grau de consumo ecológico. A hipóteses $2 \mathrm{~b}$ propôs uma relação positiva entre a idade mais elevada e o grau de consumo ecológico, e a partir dos resultados $(\beta=0,368, \mathrm{p}<0,05)$ do modelo 3 e $(\beta=0,341, \mathrm{p}<0,05)$ do modelo 5 , é possível confirmar esta hipótese.

Contudo, mesmo com um valor $(\beta=0,176, \mathrm{p}<0,05)$ do modelo 4 , a hipótese $3 \mathrm{~b}$ não pôde ser completamente confirmada, já que, no modelo 5 não se observou valor significante. Não sendo possível aceitar a hipótese que a escolaridade está positivamente relacionada com o consumo ecológico. Assim, de um total de seis hipóteses, cinco foram confirmadas.

Hipótese 1a. Existe uma influência positiva e significante do sexo feminino sobre o grau de consciência ambiental (Confirmada).

Hipótese 1b. Existe uma influência positiva e significante do sexo feminino sobre o grau de consumo ecológico (Confirmada).

Hipótese 2a. Existe uma influência positiva e significante da idade mais elevada sobre o grau de consciência ambiental (Confirmada).

Hipótese 2b. Existe uma influência positiva e significante da idade mais elevada sobre o grau de consumo ecológico (Confirmada). 
Hipótese 3a. Existe uma influência positiva e significante do nível de escolaridade sobre grau de consciência ambiental (Confirmada).

Hipótese 3b. Existe uma influência positiva e significante do nível de escolaridade sobre grau de consumo ecológico (Não-confirmada).

\section{Discussão}

Conforme a análise dos dados apresentada, foi verificada a existência de uma influência positiva e significante do sexo feminino sobre o grau de percepção de consciência ambiental e consumo ecológico. Este resultado se difere dos achados de Gilg, Barr e Ford (2005), o qual investigaram famílias no Reino Unido e não foram observadas relações significantes do sexo masculino ou feminino com o consumo verde. Da mesma forma, Molina e Durana (2003) apontam que o gênero não é variável capaz de influenciar o consumo ecológico.

Já Bertolini, Possamai e Brandalise (2011) ao investigarem o contexto brasileiro, identificaram que o sexo feminino é aquele que mais valoriza as questões ambientais. Da mesma forma, Rocha (2011) ao investigar o comportamento de consumo ecológico de cariocas, verificou que as mulheres são as mais preocupadas com os impactos ambientais em atividades cotidianas. Estes resultados são reforçados pela pesquisa de Silva et al. (2015), onde os autores também identificaram que o sexo feminino exerce influência positiva e significante sobre o comportamento ambiental.

Mesmo fora do Brasil, outras investigações apresentaram resultados consistentes com esta pesquisa. Abeliotis, Koniari e Sardianou (2010) analisaram o perfil de consumidores da Grécia confiram que, as mulheres estão mais dispostas a consumirem produtos verdes devido ao menor impacto sobre o meio ambiente. Sarkar (2011) analisou as atitudes ambientais de jovens estudantes de Bangladesh, sendo identificado que, as mulheres possuem um alto nível de comportamentos ambientais.

A maior consciência ambiental das mulheres pode fazer com que elas possuam uma maior propensão ao consumo ecológico. Destaca-se que este fenômeno pode ter em suas raízes uma forte influência dos movimentos do início dos anos 90, onde as mulheres se envolveram fortemente com as questões ligadas ao meio ambiente (DI CIOMMO, 2003; SOARES, 1998; TORNQUIS; LISBOA; MONTYSUMA, 2010). Desta forma, mesmo que alguns estudos, do início e meados da década de 2000, não apresentem diferenças entre homens e mulheres, conforme Molina e Durana (2003) e Gilg, Barr e Ford (2005), este fenômeno pode ser mais recente, conforme Rocha (2011), Sarkar (2011) e Silva et al. (2015).

A idade também foi observada como uma variável que influencia de forma positiva a consciência ambiental e o consumo ecológico. Estes resultados convergem com o estudo realizado na China por Zhaoa et al. (2014) que verificaram que a idade é uma variável determinante no comportamento e atitudes do consumidor ecologicamente consciente. Outra pesquisa realizada na Europa por Abeliotis, Koniari e Sardianou (2010) apontou que, a idade também é um fator que se relaciona diretamente com as atividades de redução de consumo, reutilização de produtos e ações de voltadas para a reciclagem de produtos.

No estudo de Gilg, Barr e Ford (2005), os autores identificaram que, entre as famílias do Reino Unido os grupos pertencentes as faixas etárias mais elevadas são os mais propensos a preferirem produtos verdes. Isto pode ocorrer, devido aos jovens, mesmo com o acesso às diversas informações sobre a necessidade de ações de manutenção do meio ambiente, não possuírem uma postura proativa em relação ao consumo ecológico e não desenvolverem uma consciência ambiental (BONNET et al., 2002).

Cabe destacar que, as relações entre a idade e consciência ambiental e consumo ecológico que foram observadas neste estudo, o qual reforça achados de estudos anteriores, são sustentadas pelos argumentos de Okamoto (1996). O autor sugere que a consciência é que conduz ao comportamento, sendo que, os mais jovens tendem a possuir um menor grau de consciência ambiental, o que reflete em seu comportamento. Steger e Meima (1998) concordam com essa proposição, destacando que existe um maior grau de entendimento sobre questões ambientais em pessoas mais velhas.

Na mesma linha, Brenton, Deniz e Stephan (2013) ressaltam que indivíduos mais velhos apresentam maior propensão ao envolvimento com ações ambientais e consumo consciente. Tais afirmações ratificam o que afirmam Pereira e Curi (2012) de que a 
relação com o meio ambiente se desenvolve a partir de percepções, pois ele se constitui como um componente da existência social. Esta visão é compartilhada por Reis, Semêdo e Gomes (2012) os quais enfatizam que a relação e o respeito à diversidade, à riqueza e a complexidade ambiental é fruto de um aprendizado que ocorre ao longo do tempo.

Diante das constatações apresentadas no estudo empírico, das proposições e percepções dos autores, pode-se cogitar que a relação com o meio ambiente a o seu complexo conjunto de fatores não se reduz ao nível e ao volume de informações que se possui. Fica evidenciado que o aprendizado, as experiências acumuladas ao longo do tempo se constituem fatores preponderantes para a construção de "consciência ambiental", refletindo-se no comportamento de consumo, justificando assim, o maior grau de consciência ambiental entre os mais velhos.

Em relação ao nível de escolaridade e sua influência sobre o grau de percepção de consciência ambiental, o observado neste estudo reforça os resultados da pesquisa de Rocha (2011). Na investigação realizada com consumidores cariocas entre os anos de 1999 e 2008, foi verificado que, quanto maior o nível de instrução maior é a preferência por ofertas de produtos ecológicos. Da mesma forma, Bertolini, Possamai e Brandalise (2011) ao analisarem uma empresa brasileira do ramo de cosméticos, identificaram que, os consumidores com maior consciência ecológica também eram aqueles que haviam concluído ou cursavam o ensino superior.

Braga Júnior, Silva e Moretti (2011) também identificaram a existência de uma maior preocupação com a redução do impacto ambiental em estudantes de pós-graduação em São Paulo. Molina e Durana (2003) já haviam efetuado um levantamento em vários países, onde também confirmaram que a educação pode proporcionar uma maior sensibilização dos indivíduos, levando-os assim, a um comportamento mais ecológico. Destaca-se, no entanto, que as relações entre nível educacional e fatores relacionados com a sustentabilidade ainda não são completamente conhecidos. Um exemplo é o estudo de Pinto, Nique, Añaña e Herter (2011) que analisaram o consumo ambientalmente responsável de água no Brasil, onde os resultados demonstraram que os consumidores verdes possuem níveis mais baixos de educação.

\section{Conclusão}

O objetivo deste artigo foi verificar a influências dos fatores demográficos sobre o grau de consciência ambiental e de consumo ecológico. Os resultados obtidos por meio de modelos de regressão múltipla confirmaram cinco das seis hipóteses formuladas, observou-se que o sexo feminino, a idade e a escolaridade possuem uma influência positiva sobre o grau de consciência ambiental, sendo o consumo ecológico afetado pelas duas primeiras variáveis.

Ainda que este estudo apresente importantes resultados ligados com os fatores demográficos e sua relação com a consciência ambiental e o consumo ecológico, cabe destacar que a investigação apresenta algumas limitações. Pode-se destacar a diversidade de variáveis de fundo comportamental que podem influenciar as percepções relacionadas com aspectos ambientais (FRAJ; MARTINEZ, 2006; ROBERTS, 1999). Conforme destacam Hambrick e Mason (1984), variáveis demográficas também podem ser proxies aceitáveis para mensuração de alguns aspectos comportamentais. Porém, questões cognitivas e valores individuais, podem ser exploradas futuramente como variáveis de controle do modelo apresentado neste estudo.

Mesmo que o poder explicativo dos modelos $\left(\mathrm{R}^{2}=\right.$ $22,5 \%)$ para consciência ambiental e $\left(\mathrm{R}^{2}=15 \%\right)$ para o consumo ecológico tenham ficando dentro dos níveis aceitáveis para estudos na área de ciências sociais, como aponta Cohen (1988), ainda é fundamental que um maior conjunto de variáveis sejam investigadas, afim de se obter modelos mais robustos em termos de poder explicativo. Sugere-se que futuras pesquisas avancem neste sentido, identificando um maior conjunto de variáveis demográficas para melhoria do modelo.

Apesar de uma amostra expressiva para este tipo de estudo, 1075 respondentes, e também da utilização da região de moradia - centro / periferia - como uma das variáveis de controle, o estudo foi realizado com respondentes de um único município da região Sul do Brasil. Assim, futuras investigações podem também utilizar variáveis de fundo cultural como variável de controle, possibilitando uma visualização mais clara sobre diferenças culturais. Mesmo que, os resultados encontrados não possam ser generalizados, o estudo auxilia no melhor entendimento das variáveis 
demográficas e sua relação com o consumo ecológico e a consciência ambiental.

Mesmo que os aspectos demográficos não sejam os únicos que possam influenciar os níveis de consciência ambiental e o consumo ecológico, o estudo fornece importantes indícios sobre a relação destas variáveis. Como principais contribuições destaca-se a identificação de que as mulheres venham se destacando como as mais preocupadas com o meio ambiente, diferentemente do que ocorria até início dos anos 2000. As experiências, aprendizado e conhecimentos acumulados com a idade também parece ser um fator determinante no aumento da consciência ambiental e consumo ecológico. Por fim, a escolaridade ainda é uma variável que merece aprofundamento, pois o próprio contexto e tipo de formação podem ocasionar interferir na percepção das questões ambientais.

\section{Referências}

ABELIOTIS, K.; KONIARI, C.; SARDIANOU, E. The profile of the green consumer in Greece. International Journal of Consumer Studies. v. 34, n. 2, p. 153-160, 2010. https://doi.org/10.1111/ j.1470-6431.2009.00833.x

BALDERJAHN, I. Personality variables and environmental attitudes as predictors of ecologically responsible consumption patterns. Journal of Business Research, v.17, p.51-56, 1988. https:// doi.org/10.1016/0148-2963(88)90022-7

BERTOLINI, G. R. F; POSSAMAI, O. Proposta de instrumento de mensuração do grau de consciência ambiental, do consumo ecológico e dos critérios de compra de consumidores. Revista de Ciência \& Tecnologia. v.13. n. 25/26, p. 19-27, 2005.

BERTOLINI, G. R. F; POSSAMAI, O.;

BRANDALISE, L. T. A percepção dos consumidores de produtos ecologicamente corretos: estudo de caso em pequena empresa. Revista da Micro e Pequena Empresa, v. 3, n. 1, p. 99-119. 2011.

BONNET, J. F.; DEVEL, C.; FAUCHER, P.; ROTURIER, J. Analysis of electricity and water end-uses in university campuses: case-study of the University of Bordeaux in the framework of the Ecocampus European Collaboration. Journal of
Cleaner Production, v. 10, n. 1, p. 13-24, 2002. https://doi.org/10.1016/s0959-6526(01)00018-x

BRAGA-JÚNIOR, S. S.; SILVA, D.; MORET'TI, S. L. A. Fatores de influência no consumo "verde": um estudo sobre o comportamento de compra no setor supermercadista. Revista Brasileira de Marketing, São Paulo, v. 10, n. 1, p 151-176, 2011. https://doi. org/10.5585/remark.v10i1.2213

BRANDALISE, L. T.; SILVA, J. M. RIBEIRO, I.; BERTOLINI, G. R. F. O reflexo da disciplina de educação ambiental na percepção e conduta dos universitários. Pretexto, v. 15, n. 4, p. 11-26, 2014.

BRENTON, M. W.; DENIZ S. O.; STEPHAN, D. Age and environmental sustainability: a metaanalysis. Journal of Managerial Psychology, v. 28, v. 7/8, p. 826-856, 2013. https://doi.org/10.1108/ jmp-07-2013-0221

BRUNDTLAND, G. H. Nosso futuro comum. Rio de Janeiro: FGV, 1987.

BRÜSEKE, F. J. O problema do desenvolvimento sustentável. Desenvolvimento e natureza: estudos para uma sociedade sustentável. São Paulo: Cortez, 1995.

CAMPONOGARA S.; RAMOS F. R. S.; KIRCHHOF A. L. C. Reflexivity, knowledge and ecological awareness: premises for responsible action in the hospital work environment. Revista Latino Americana de Enfermagem, v.17, n. 6, p. 1030-1036, 2009. https:/ / doi.org/10.1590/s010411692009000600016

CARVALHO, A. O.; CIRANI, C. B. S.; RIBEIRO, I.; CINTRA, R. F. Viabilidade econômica e sustentabilidade, relações antagônicas ou complementares? Revista Competitividade e Sustentabilidade, v. 2, n. 1, p. 63-75, 2015. https:// doi.org/10.5935/2359-5876.20150005

COHEN, J. Statistical Power Analysis for the Behavioral Sciences. New York: Psychology Press, 1988.

CONNELLY, B. L.; KETCHEN JR., D. J.; SLATER, S. F. Toward a "theoretical toolbox" for sustainability research in marketing. Journal of the Academy of Marketing Science, v. 39, n. 1, p. 86-100, 2011. https://doi.org/10.1007/s11747-010-0199-0 
CÔRTES, P. L.; DIAS, A. G.; FERNANDES, M.

E. S. T.; PAMPLONA, J. M. V. Environmental behavior: a comparative study between Brazilian and Portuguese students. Ambiente \& Sociedade, v. 19, n. 3, p. 113-134, 2016. https://doi. org/10.1590/1809-4422asoc139099v1932016

COSTA, G. Atitudes e comportamentos das famílias sobre consumo sustentável. 2011. 178 F, Dissertação (Mestrado em Cidadania Ambiental e Participação) - Universidade Aberta de Portugal, Lisboa, 2011.

DAL BEN, J. C. R.; MELO, C. M.; FIGUEIRAS, G. B. Análise do comportamento e sustentabilidade: revisão dos artigos publicados no Behavior and Social Issues de 2005 a 2016. Revista Brasileira de Análise do Comportamento, v. 12, n. 2, p. 86-94, 2016. https://doi.org/10.18542/rebac.v12i2.4401

DI CIOMMO, R. C. Relações de gênero, meio ambiente e a teoria da complexidade. Revista de Estudos Feministas, v. 11, n. 2, p. 423-443, 2003. https://doi.org/10.1590/s0104-026x2003000200005

EAGLY, A. H. Sex differences in social behavior: A social-role interpretation. Hillsdale, New Jersey: Lawrence Erlbaum, 1987.

ENGEL, J. F.; BLACKWELL, R. D.; MINIARD, P. W. Consumer behavior. Orlando: The Dryden Press, 1995.

FERREIRA, M. C. Gestão Ambiental: um estudo em empresas do setor sucroalcooleiro em goiás. Revista Pensamento Contemporâneo em Administração, v. 7, n. 3, p 59-80, 2013. https://doi.org/10.12712/ rpca.v7i3.264

FRAJ, E.; MARTINEZ, E. Influence of personality on ecological consumer behaviour. Journal of Consumer Behaviour, v. 5, n. 3, p. 167-181, 2006. https://doi.org/10.1002/cb.169

GILG, A.; BARR, S.; FORD, N. Green consumption or sustainable lifestyles? Identifying the sustainable consumer. Futures. v. 37. n. 6, p. 481-504. 2005. https://doi.org/10.1016/j.futures.2004.10.016

GORNI, P. M.; GOMES, G.; DREHER, M. T. Consciência ambiental e gênero: os universitários e o consumo sustentável. Revista de Gestão Social e Ambiental, v. 6, n. 2, p. 165-179, 2012. https://doi. org/10.24857/rgsa.v6i2.468
GUIMARÃES, M.; OLABARRIAGA, N.;

TONSO, S. A pesquisa em políticas públicas e educação ambiental. Pesquisa em Educação Ambiental, v. 4, n. 2, p. 215-227, 2009. https://doi. org/10.18675/2177-580x.vol4.n2.p215-227

HAMBRICK, D. C.; MASON, P. A. Upper

Echelons: The organization as a reflection of its top managers. Academy of Management Review, v.

9, n. 2, p. 193-206, 1984. https://doi.org/10.5465/ amr.1984.4277628

HAMES C.; FRISON M. D.; ARAÚJO M. C. P. A Educação ambiental como articuladora na produção de saberes e no desenvolvimento da consciência ambiental. Revista eletrônica do Mestrado Educação Ambiental. v. 23, n. S1, p. 88-102, 2009.

ISERHARDT, P. M.; PEREIRA, L. P.; MACHADO, E. BONELLA, S. D. Consciência ambiental: a melhor forma de sobrevivência. In: X Salão de Iniciação Científica - PUCRS. Anais... 2009, p. 2493-2495.

JABBOUR, C. Gestão Ambiental em Escolas de Negócios: mapeando o estado da arte. Revista Pensamento Contemporâneo em Administração, v. 8, n. 4, p. 1-22, 2014. https://doi.org/10.12712/ rpca.v8i4.461

JACOBI, P. Educação ambiental, cidadania e sustentabilidade. Cadernos de Pesquisa, n. 118, p. 189-205, 2003. https://doi.org/10.1590/s010015742003000100008

KATES, R.; PARRIS, T.; LEISEROWITZ, A. What is sustainable development? Goals, indicators, values, and practice. Environment: Science and Policy for Sustainable Development, v. 47, n. 3, p. 8-21, 2005. https://doi.org/10.1080/00139157.2005.10524444

LAROCHE, M.; BERGERON, J.; BARBAROFORLEO, G. Targeting consumers who are willing to pay more for environmentally friendly products. Journal of consumer marketing, v. 18, n. 6, p. 503-520, 2001. https://doi.org/10.1108/ eum0000000006155

LARSON, L. R.; STEDMAN, R. C.; COOPER, C. B.; DECKER, D. J. Understanding the multidimensional structure of pro-environmental behavior. Journal of Environmental Psychology, v. 43, p.112-124, 2015. https://doi.org/10.1016/j. jenvp.2015.06.004 
LEVINE, D. S.; STRUBE, M. J. Environmental attitudes, knowledge, intentions and behaviors among college students. Journal of Social Psychology, v. 152, n. 3, p. 308-326, 2012. https:// doi.org/10.1080/00224545.2011.604363

LOPES, V. N.; PACAGNAN, M. N. Marketing verde e práticas socioambientais nas indústrias do Paraná. Revista de Administração, v. 49, n. 1, p. 116-128, 2014. https://doi.org/10.5700/rausp1135

MARIGA, J. T. Educação e meio ambiente. Revista Ciências Sociais em Perspectiva, v. 8, n. 5, p. 139145, 2006.

MENDES, J. M. G. Dimensões da sustentabilidade. Revista das Faculdades Santa Cruz, v. 7, n. 2, p. 49-59, 2009.

MOISANDER, J. Motivational complexity of green consumerism. International Journal of Consumer Studies, v. 31, n.4, p. 404-409, 2007. https://doi. org/10.1111/j.1470-6431.2007.00586.x

MOLINA, M. A. V.; DURANA, C. A. G. Aproximación al perfil sociodemográfico del consumidor ecológico a través de la evidencia empírica: propuestas para el desarrollo del mix de marketing. Boletín Económico de Ice. n. 2777, p. 25-40, 2003.

MOREIRA, L. A. S. Sustentabilidade Ambiental: Avanço ou retrocesso para desenvolvimento. Editora Clube de Autores, 2015.

OKAMOTO, J. Percepção ambiental e comportamento. São Paulo: Plêiade, 1996.

ONU. Declaração de Joanesburgo sobre desenvolvimento sustentável. Disponível em: http://www.onu.org.br/rio20/documentos/

PAÇO, A. M. F.; RAPOSO, M. L. B. Green consumer market segmentation: empirical findings from Portugal. International Journal of Consumer Studies, v. 34, n. 4, p. 429-436, 2010. https://doi. org/10.1111/j.1470-6431.2010.00869.x

PEÇANHA, R. S.; IIZUKA, E. S. Análise da produção científica brasileira sobre sustentabilidade entre os anos de 2008 a 2011. Revista de Gestão Ambiental e Sustentabilidade, v. 3, n. 1, p. 1-17, 2014. https://doi.org/10.5585/geas.v3i1.80
PEREIRA, S. S.; CURI, R. C. Meio ambiente, impacto ambiental e desenvolvimento sustentável: conceituações teóricas sobre o despertar da consciência ambiental. Revista de Administração, Contabilidade e Sustentabilidade, v. 2, n. 4, p.3557, 2012. https://doi.org/10.18696/reunir.v2i4.78

PINTO, D. C.; NIQUE, W. M.; AÑAÑA, E. S.; HERTER, M. M. Green consumer values: how do personal values influence environmentally responsible water consumption? International Journal of Consumer Studies, v. 35, n. 2, p. 122-131, 2011. https://doi.org/10.1111/j.14706431.2010.00962.x

REIS, L. C. L.; SEMÊDO, L. T. A. S.; GOMES, R. C. Conscientização ambiental: da educação formal a não formal. Revista Fluminense de Extensão Universitária, v. 2, n. 1, p. 47-60, 2012.

ROBERTS, J. A.; BACON, D. R. Exploring the subtle relationships between environmental concern and ecologically conscious consumer behavior. Journal of Business Research, v. 40, n. 1, p. 79-89, 1997. https://doi.org/10.1016/s01482963(96)00280-9

ROCHA, A. L. Intenções e ações em relação a escolhas de produtos ecológicos: estudo sobre o comportamento do consumidor carioca. Revista Contemporânea de Economia e Gestão. v. 9, n. 1, 2011.

SARKAR, M. Secondary students' environmental attitudes: the case of environmental education in Bangladesh. International Journal of Academic Research in Business and Social Sciences, v. 1, p. 106-116, 2011.

SCHIFFMAN, L. G.; KANUK, L. L.

Comportamento do consumidor. Rio de Janeiro: LTC, 2000.

SEQUINEL, M. M. C. C. M. M.; CARON, A. Consumo sustentável: uma questão educacional e de múltiplas reponsabilidades. Revista Brasileira de Ciências Ambientais, v. 16, p. 36-43, 2010. 
SILVA, A. M.; MEIRELES, F. R. S.; REBOUÇAS, S. M. D. P.; ABREU, M. C. S. Comportamentos ambientalmente responsáveis e sua relação com a educação ambiental. Revista de Gestão Ambiental e Sustentabilidade, v. 4, n. 1, p. 1-16, 2015. https:// doi.org/10.5585/geas.v4i1.205

SILVA, A. M. M.; LOPES, W. G. R. O Consumo Responsável e o Círculo Virtuoso do Marketing Verde. Espacios. v. 36, n. 21, p. 17, 2015.

SOARES, B. E. C.; NAVARRO, M. A.; FERREIRA, A. P. Desenvolvimento sustentado e consciência ambiental: natureza, sociedade e racionalidade. Ciências \& Cognição, v. 02, p. 42-49, 2004.

SOARES, V. Muitas faces do feminismo no Brasil. In: Borba, Â.; Faria, N.; Godinho, T. (Orgs.). Mulher e política: gênero e feminismo no partido dos trabalhadores. São Paulo: Fundação Perseu Abramo, 1998.

SOLOMON, M. R. Consumer behavior: buying, having and being. New Jersey: Prentice-Hall, 1996.

SPÍNOLA, A. L. Consumo sustentável: o alto custo dos produtos que consumimos. Revista de Direito Ambiental, v. 6, n. 24, p. 209-216, 2001.

STEG, L.; VLEK, C. Encouraging proenvironmental behaviour: an integrative review and research agenda. Journal of Environmental Psychology, v. 29, p. 309-317, 2009. https://doi. org/10.1016/j.jenvp.2008.10.004

STEGER, U.; MEIMA, R. The strategic dimensions of environmental management: sustaining the corporation during the age of ecological discovery. London: Macmillan, 1998.

STERN, P. C.; DIETZ, T.; GUAGNANO,

G. A. The new ecological paradigm in socialpsychological context. Environment and behavior, v. 27, n. 6, p. 723-743, 1995. https://doi. org/10.1177/0013916595276001

TORNQUIST, C. S.; LISBOA, T. K.;

MONTYSUMA, M. F. Mulheres e meio ambiente.

Revista Estudos Feministas, v. 3, n. 18, p. 865-869, 2010.

YOUNG, W.; HWANG, K.; McDONALD, S.; OATES, C. J. Sustainable consumption: green consumer behaviour when purchasing products.
Sustainable Development, v. 18, n.1, 20-31, 2010. https://doi.org/10.1002/sd.394

ZHAOA, H.; GAOB, Q; WUA, Y.; WANGA, Y.; ZHUA, $\mathrm{X}$. What affects green consumer behavior in China? A case study from Qingdao. Journal of Cleaner Production, v. 63, n. 15, p. 143-151, 2014. https://doi.org/10.1016/j.jclepro.2013.05.021 